\section{Ingeniería}

CONTENIDO

Artículos

Análisis numérico de un sistema de aire acondicionado empleando
mallas estructuradas y no estructurades A

Funciones de regresión para caudales extremos en la Vertiente Rojas, Nazareth; Aguilar, José Francisco; Solís, Hernán. Evaluación de daños por agresión ambiental en viviendas
de concreto reforzado. Solís, Rómel; Moreno, Eric; Jiménez, Felipe; Rosas, Victorino.

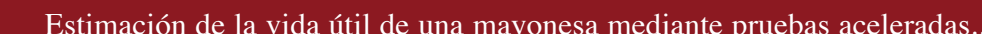
Estimación de la vida útil de una mayonesa
García, Claudia; Molina, Manuel.

Inestabilidad oscilatoria de tensión debido a los motores de inducción …….....................65-75 rde, Gustavo; Araya, Eddie.

Estabilidad y colapso de tensión en sistemas eléctricos Araya, Eddie.

Obtención de pulpa a la soda antraquinona de Tectona grandis creciendo en Costa Rica...........................

Protocolo evaluador para el manejo de desechos y la seguridad ocupacional
aplicado al laboratorio clínico del Hospital San Francisco de Asís de Grecia. Ruiz, Francisco.

9. Euler: su contex
Vargas, Celso.

10. Reserva de resistencia de edificios porticados de concreto armado disenaados conforme al ACI-318/ IBC 2006 ........ Análisis y comentarios

Herrera, Rodolfo.

breve semblanza.

Trabajos de Graduación 2007

Lista de proyectos de graduación de grado y posgrado

Normas

$\sum_{\text {EDTORAL }}$

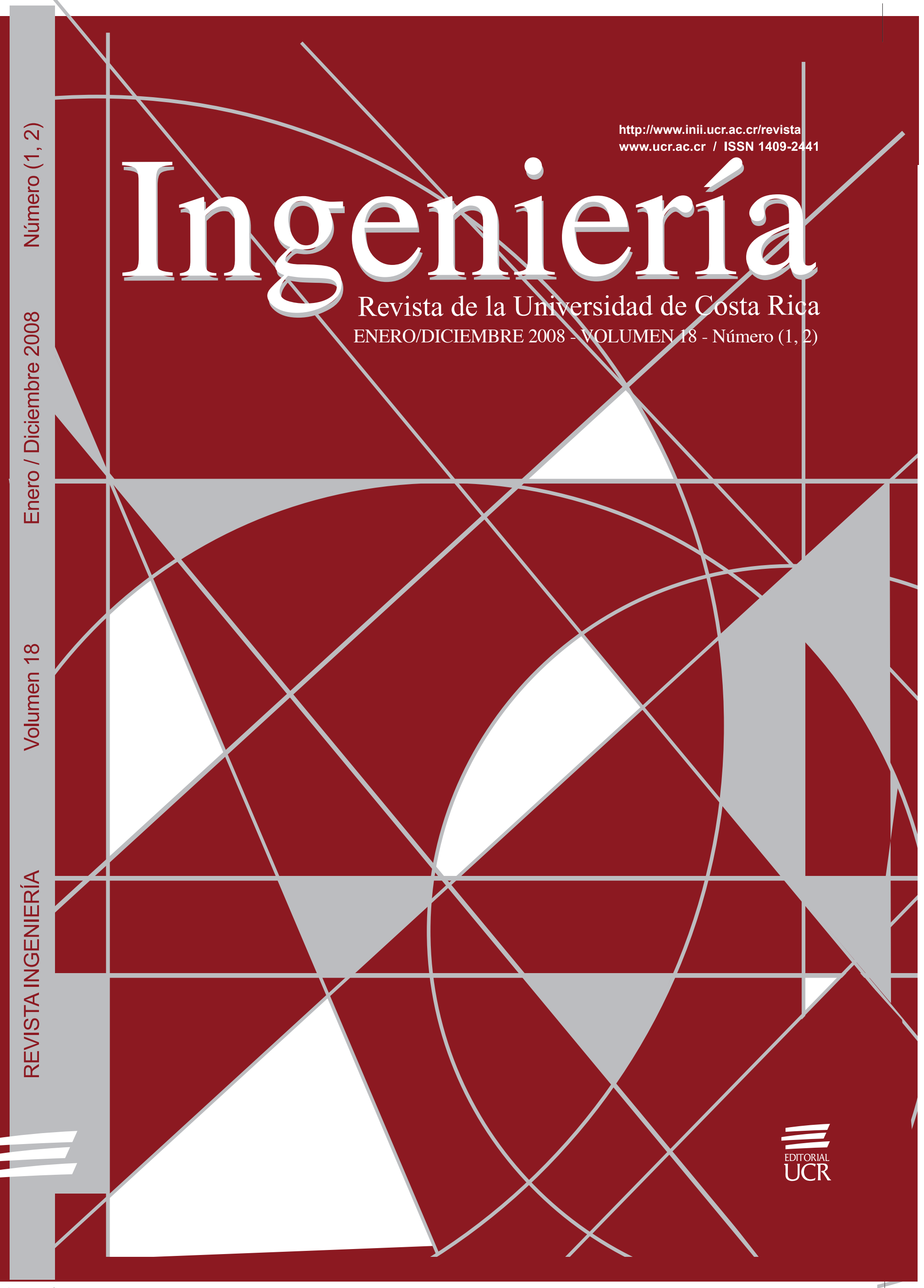




\title{
EULER: SU CONTEXTO MATEMÁTICO, FILOSÓFICO Y CIENTÍFICO
}

Celso Vargas Elizondo

\begin{abstract}
Resumen
Berhnard Euler fue un pensador de una gran capacidad y creatividad. Algunos de sus trabajos son verdaderas piezas de ingenio. Todos admiramos la solución que ofrece al problema de los puentes de Köningsberg, quizá una de sus obras más populares, en la que uno de es llevado paso a paso hacia diferentes alternativas y soluciones cada vez más generales. Sin embargo, al igual que todo el mundo es hijo de su época, y enfrentó una serie de problemas que, por las herramientas conceptuales existentes le imposibilitaron ir más allá en la aplicación de su ingenio. En particular, la formalización del cálculo infinitesimal debió esperar alrededor de un siglo hasta que, nuevos desarrollos y la distancia que proporciona el tiempo, permitieron a un Cauchy y a otros grandes matemáticos darle una formulación rigurosa y clara que lo posicionó como uno de los campos más importantes de las matemáticas modernas. Sin embargo, como ocurre casi siempre, los aportes de Cauchy no ignoran, sino que incorporan lo alcanzado hasta el momento. Podemos parafrasear, para el caso que comentamos, lo que Newton dijera en relación con Galileo, Kepler y otros: "he estado sobre hombres de gigantes". Sin ninguna duda, Euler es uno de estos gigantes.

Este trabajo se divide en tres temas principales: la ubicación de Euler en el desarrollo de las matemáticas; lo que podría denominarse con Berkson, "la situación problemática de Euler", es decir, las principales visiones y las críticas al cálculo infinitesimal que Euler tuvo que sopesar. El tercero, las contribuciones principales de Euler al desarrollo del cálculo.
\end{abstract}

Palabra clave: Euler, cálculo infinitesimal, historia de las matemáticas, Leibniz, Newton

\begin{abstract}
Berhnard Euler is one of the thinkers of most creativity and depth. He played one important role in the development of infinitesimal calculus. From a historical perspective we can situate him in the transition period between the invention of calculus by Newton and Leibniz during the second half of XVII century and its rigorous formulation by Cauchy and other contemporary mathematicians. In this sense, Euler made meaningful contribution to transit from a logical approach to a more functional one. In the context of three hundred year of his birthday, we analyze Euler's contributions to the calculus. Euler engaged in different fields of knowledge and engineering, but we limit ourselves to the development of calculus. The paper divides into three sections. The first one, very brief, situates Euler in the context of the development of calculus; the second discussion detailed the problematic situation of Euler and the third one, his contributions to this important field of mathematics.
\end{abstract}

Key words: Euler, infinitesimal calculus, history of mathematics, Leibniz, Newton

Recibido: 20 de agosto del 2008 • Aprobado: 16 de setiembre del 2008

\section{EULER Y SU UBICACIÓN EN EL DESARROLLO DE LAS MATEMÁTICAS}

Si seguimos la clasificación de las etapas en el desarrollo del cálculo infinitesimal propuestas por Boyer (1959), los aportes de Euler se ubican en el periodo denominado de indecisión, es decir, aquel periodo de transición entre la invención del cálculo, de manera independiente, por Newton y Leibniz y la etapa en la que el cálculo infinitesimal recibe su formulación rigurosa durante el siglo XIX gracias a los trabajos de un grupo realmente importante de matemáticos, incluyendo a Cauchy, 
Bolzano, Gauss, Riemann, Abel, Cantor y otros En efecto, no fue sino hasta el advenimiento de la teoría moderna de conjuntos y las investigaciones sobre las funciones, cuando se le da un tratamiento riguroso al cálculo infinitesimal. En este sentido, recordamos el análisis realizado por Frege en las postrimerías del siglo XIX en el que definía las funciones a partir de dos características: eran no saturadas y necesitadas de completación. Durante la primera mitad del siglo XX ya encontramos la formulación actual, gracias a los trabajos de Church y otras personas. Lo anterior no significa que no se avanzara significativamente durante los siglos anteriores. Lo que indicamos es que determinados desarrollos potencian la integración de resultados que se habían o venían alcanzando. Es como las piezas de un rompecabezas, de pronto algunos desarrollos permiten armarlo de una manera más armoniosa.

Vamos a ver que Euler anticipa algunos aspectos sobre el cálculo, lo cual lo ubica como uno de los antecesores del tipo de formalización que encontramos a final del siglo XIX, junto a David Bernoulli, Lagrange y otros distinguidos matemáticos. Sin embargo, los aportes de Euler no se limitan, como es usual, al cálculo sino que también conciernen con temas de mecánica, óptica y astronomía. Se dice que fue el primero en desarrollar una dinámica de partículas newtonianas sobre la base de los métodos analíticos, establecimiento un conjunto de ecuaciones generales sobre el movimiento de partículas, tomando el movimiento de un cuerpo sólido a partir de su centro de masa. Pero también incursiona en otras ramas de las matemáticas como la teoría de los números y el análisis matemático. Finalmente, se ocupó de asuntos prácticos relacionados con la navegación y con los sistemas de agua.

La preocupación por temas físicos en todos estos pensadores pone de manifiesto, por un lado, la manera cómo se originó el cálculo: para resolver problemas relacionados con distintos tipos de movimiento. Pero por el otro lado, el gran atractivo de la física para todos los intelectuales de la época, gracias a la revolución newtoniana. De hecho esta fue la razón por la cual costó mucho que los matemáticos se dieran cuenta de que el método infinitesimal puede ser formalizado como una teoría independiente de los fenómenos físicos que motivaron su desarrollo. Aunque, como veremos, la situación es mucho más compleja que esto: hay una serie de posturas metafísicas y también metodológicas que condicionaron la posibilidad de darle una formulación matemática precisa al cálculo.

\section{LA SITUACIÓN PROBLEMÁTICA DE EULER}

Nos interesa ubicar aquí lo que podríamos denominar, tomando prestada una frase de Berkson, "la situación problemática de Euler". Es a partir de este trasfondo que podemos apreciar los problemas y las contribuciones de Euler. En este caso, nos situamos en la formulación del cálculo tal y como lo hicieron Newton y Leibniz, y los problemas filosóficos y metodológicos que surgen a partir de ahí. Sí debemos advertir que el establecimiento de puntos absolutos de partida no se justifica ni filosófica ni históricamente. Problema similar se presenta cuando tratamos de buscar antecedentes para un enfoque. El establecimiento de los límites temporales es un problema importante y claramente no es el lugar siquiera de plantearlo.

\subsection{Newton y Leibniz}

Newton y Leibniz llegaron de manera independiente al método general para resolver problemas relacionados con la cuadratura (integrales) y las tangentes (derivadas). Fueron ellos quienes visualizaron que uno es el inverso del otro, y propusieron métodos para pasar de las tangentes a las cuadraturas y viceversa, mostrando que el uno es el inverso del otro.

Fue durante la década de 1660-1670 cuando Newton llegó al procedimiento general para resolver este tipo de problemas. Sin embargo, la publicación de su método se realizó en el siglo siguiente. Por su parte, Leibniz estableció su método en 1675 (Hall, 2002). Claramente, existen similitudes y diferencias entre ambos intelectuales. Entre las similitudes está el hecho de que ambos eran protestantes y provenían 
de familias protestantes; además, ambos compartieron una determinada concepción mecánica del mundo. Pero existen diferencias fundamentales (de índole metafísica) entre ambos enfoques, lo que marcó diferencias en la promoción y extensión del método a nuevas áreas de la matemática más allá de las del movimiento, es decir, de la mecánica.

\subsubsection{Newton}

Newton, como ha señalado Buckley (2003) trató de fundamentar tanto las matemáticas como la teología en la mecánica. Newton se declara continuador de la tradición de Arquímedes, y sobre todo de Pappus de Alejandría, quien habló de una mecánica universal. Newton es un renovador de esta concepción y se opone a la división escolástica del conocimiento dominante en su época. En efecto, como ha señalado Garbey (2002), la filosofía en ese momento se dividía en práctica y especulativa. La especulativa en tres ciencias principales: la metafísica o filosofía primera, la filosofía natural y la matemática. La filosofía natural es la ciencia de las causas de los cambios y del estado actual de mundo. La matemática es la ciencia de los números, de la extensión y de la medida, la cual se obtiene por abstracción de las cosas materiales. Dentro de este enfoque, claramente, como en Descartas, la filosofía primera es la fundamental en la construcción del universo del conocimiento.

Esta visión es la que es profundamente cuestionada por Newton. Antepone la mecánica, como punto de partida del conocimiento. Newton, como ha puesto de manifiesto Gabey (2002) no tuvo una sola noción de "mecánica". Tampoco define el alcance de esta de manera precisa. Pero, parece haber seguido como heurística el que la mecánica mantiene cierta relación de isomorfismo con un tratamiento matemático de los fenómenos. Pero no cualquier tipo de aproximación matemática, sino aquella que sea justificada desde el punto de vista físico.

Pero el programa general de Newton va más allá: busca ampliar el ámbito de la filosofía natural de manera que incluya, tanto la mecánica como la teología, como ya hemos mencionado. En el
Escolio General a los Principia Mathematica de Philosophia Naturalis, encontramos una serie de preguntas que permiten visualizar, según Newton, en un solo marco las tareas de la nueva filosofía natural:

¿Qué hay en los lugares casi vacíos de materia; y de dónde es que el Sol y los planetas gravitan el uno hacia el otro, sin que haya materia densa entre ellos?¿De dónde es que la naturaleza no hace nada en vano; y de dónde surge todo ese orden y belleza que vemos en el mundo?¿Con qué fin existen los cometas y de dónde es que los planetas se mueven todos de la misma manera en órbitas concéntricas, mientras que los cometas se mueven de todas las formas posibles en órbitas muy excéntricas; y qué impide a las estrellas fijas caer unas sobre otras?

¿Cómo llegaron los cuerpos de los animales a ser inventados con tanto arte, y con qué fin sus varias partes?¿Fue el ojo inventado sin habilidad en óptica, y el oído sin conocimiento de los sonidos? ¿Cómo es que los movimientos del cuerpo siguen a la voluntad, y de dónde el instinto en los animales?¿No es la sensibilidad de los animales la que ubica qué substancia sensitiva está presente, y mediante qué especie sensible de las cosas debe ser llevada a través de los nervios y el cerebro, que pueden percibir por su inmediata presencia a esta substancia? (Citado en Buckley, 2003: 103).

Por un lado, entonces, está el rango tan amplio de la Mecánica: todos aquellos fenómenos que son susceptibles de ser abordados de manera consistente desde el punto de vista del nuevo método: las matemáticas surgiendo de la mecánica. Pero, por otro lado, lo que es también fundamental es que las preguntas que se plantean a la mecánica obligan a que se busque una causa primera, la cual no puede ser mecánica, a saber, Dios. La mecánica no es teología, pero es su fundamento. El diseño del mundo, la perfección de lo creado, la simplicidad de los principios racionales con los 
que fue creado, muestran de manera inequívoca la existencia de un Ser Todopoderoso, eterno, infinito, absolutamente perfecto, omnipresente y omnisciente. Pero lo más importante es que "(su) substancia nos es desconocida; conocemos a Dios solamente a través de sus atributos y la excelencia del orden natural, y a través de las causas finales de las cosas" (Garbey, 2002: 332).

McClaurin, el matemático y discípulo de Newton, presentando el programa newtoniano, vio a la "filosofía natural" como el más firme bastión contra el ateísmo, asegurando la religión natural igualmente contra la vana sofistería de los Epicuros y los peligrosos refinamientos de los metafísicos (Garbey, 2002: 333).

\subsubsection{El cálculo newtoniano}

La aproximación newtoniana al cálculo se da mediante la geometría. De hecho, todos sus ejemplos son modelados en términos geométricos. Los Principia, en los que Newton hace uso extensivo _aunque implícito_ del cálculo, es de manera geométrica. En este sentido, Newton es fundamentalmente un geometra. Así lo reconoce Hall (2002:432) "No es coincidencia que el único conjunto de lecturas de Newton sobre Álgebra fueron publicados bajo el titulo de Arithmética Universals. (...). Posteriormente, en el curso de su larga disputa con Leibniz y con los matemáticos continentales, Newton mantuvo que (similar a los antiguos geometras), después de haber encontrado las proposiciones de los Principia por análisis, los ha demostrado al lector por medio de la geometría". La geometría ocupaba un lugar importante para Newton, pues se ajustaba mucho mejor a su programa metafísico, es decir, ponía de manifiesto ciertas propiedades del Espacio Absoluto que, nuevamente Newton asoció con el "receptáculo de Dios".

Por otro lado, la manera de conceptualizar el cálculo pone de manifiesto su posición de que las matemáticas derivan de la mecánica. En efecto, Newton habla de fluxiones y de fluentes para referirse a los componentes involucrados en la modelación del movimiento. El primero refiere a la tasa de variación del movimiento en un momento determinado, mientras que el segundo a la suma (ratios) de esas variaciones. Son estas razones las que pueden sufrir incrementos o decrementos lo que permite estimar su convergencia hacia un valor en función del tiempo, es decir, su límite. El método de las fluxiones, que es el problema general del cálculo, consiste en encontrar a partir de determinadas cantidades dadas, la razón de la variación de éstas. Como puede observarse, la manera de formularlo está muy fuertemente condicionada a problemas físicos. Como señala Mahoney (1998:723) “A pesar de la legenda, Newton no creó las fluxiones para acomodar problemas que involucran movimiento. Por el contrario, como lo muestra desde el primer ensayo de lo que sería la técnica de las fluxiones, él comenzó con el movimiento, siguiendo así una línea de pensamiento que hunde sus raíces en fuentes clásicas pero dándole una nueva vitalidad por medio de los desarrollos de la mecánica (...)”.

Además del uso de una notación confusa y poco práctica que consistía en indicar los pequeños incrementos con el 0 (no el cero), Newton identifica la fluxión con el momentum, lo que llevará a una serie de problemas de interpretación. Para comenzar, como señala Mahoney, el concepto de "momento" presenta una ambigüedad que solo fue superada más tarde. "Momento" significa tanto un instante de tiempo como "la fuerza mediante la cual un sistema mecánico está en equilibrio". Segundo, el concepto de "pequeño incremento" y "anulación del incremento" implicado en la determinación de la tasa de variación no resultaba nada claro de interpretar. En tercer lugar, estos infinitesimales resultaban realmente difíciles de interpretar desde el punto de vista físico. Aceptar un punto de vista realista, es decir, que la naturaleza es infinitamente indivisible planteaba problemas importantes sobre la justificación de las unidades de medida que debemos adoptar para captar este aspecto de la naturaleza. Pero si eran ficciones, esto dejaba mal parado el asunto de la relación entre mecánica y matemática, base al pensamiento newtoniano.

Estos problemasnoeran exclusivos deNewton, sino también de la otra posición, la continental, iniciada con Descartes, continuada por Leibniz y que lleva finalmente a la formulación del cálculo por Cauchy y otros. Veamos brevemente la "acera del frente". 


\subsubsection{Leibniz}

Leibniz proviene de las humanidades, de la lógica, de la metafísica, de la teología y del derecho. Esta es su formación básica. La aproximación que hace Leibniz de la física y del nuevo cálculo está fuertemente influida por esta visión humanista previa. Las críticas que hace a la física newtoniana y a la cartesiana están relacionadas con lo que él consideró como una inadecuación "de la concepción mecánica del mundo" respecto de las características propias de la vida y el pensamiento. Para él, la física no es ni debe ser extraña a la vida y a los fenómenos psicológicos. Una visión general debe proporcionar principios unificadores tanto para la física, la biología y la psicología. Se esforzará durante toda su vida por establecer este marco unificador. Su principal estrategia será la lógica. Como señala Wiener (1951) la lógica tenía para Leibniz dos funciones básicas: por un lado, servir a propósitos teológicos para demostrar la existencia de Dios; por el otro, cumplir el papel clave de encontrar fundamentos para la ciencia, el trabajo ingenieriel y el orden social. Leibniz nunca separó el ámbito teórico de la actividad práctica y de la visualización y construcción de una sociedad o de un mundo mejor.

La lógica, esta herramienta conceptual, es denominada "arte combinatorio", un arte de combinar conceptos de manera simbólica mediante el cual se podían establecer distintas relaciones y representar diferentes conceptos, tanto abstractos como concretos. En una carta enviada por Leibniz a Herr Johann Friedrich en 1671 , relata como este instrumento le ha permitido abordar los más diversos temas y establecer las relaciones de deducción que se derivan de estos campos. Llegó a afirmar que en el futuro las disputas se resolverían mediante el cálculo.

El Arte Combinatorio estaba constituido por dos partes. Un conjunto de caracteres primitivos que representaban conceptosirreductibles, esdecir, conceptos simples. Este componente constituía el alfabeto del arte. El segundo componente era el conjunto de reglas para reducir ideas o conceptos de complejos a simples. Sin embargo, este conjunto de reglas funcionaba también a la inversa. Partiendo de conceptos simples podemos formar conceptos cada vez más complejos. El proceso de construcción o de reducción puede recorrerse en ambas direcciones. Así podemos exhibir diferentes relaciones entre los elementos, como las series matemáticas y otros arreglos que comparten una determinada propiedad o, mejor dicho, son captadas bajo un solo concepto.

Leibniz no cree que todo pueda ser reducido a conceptos simples de manera lógica, es decir, que sea clara la manera en qué un concepto se reduce a un conjunto de primitivos. Esto le lleva a la famosa distinción entre verdades de hecho y relaciones de ideas. Para las primeras tenemos incapacidad de mostrar las relaciones lógicas de deducibilidad mediante las cuales se relacionan sus conceptos. Pero no es una limitación para Dios. De acuerdo con Leibniz, las proposiciones que podemos componer se agrupan en varias categorías, en particular tres: los axiomas, las definiciones y aquellas proposiciones que expresan relaciones de hecho. Los axiomas, como veremos más adelante, jugarán un papel muy importante en su análisis de las matemáticas.

La idea de que existen primitivos irreductibles a otros conceptos, le llevó a adherir el principio de composición, tan fundamental en la lógica moderna. Sin embargo, se encontró con una serie de problemas, siendo el principal de ellos, las limitaciones que ofrecía el que una oración debe expresarse en términos de sujeto-predicado. Otra cosa hubiera sido si contara con las poderosas herramientas de formalización lógica del siglo $\mathrm{XX}$. Adicionalmente, encontró serios problemas para precisar el conjunto de primitivos que forma el alfabeto del lenguaje. Leibniz se encuentra con que hay infinito número de estos primitivos ("la naturaleza es infinitamente compleja, en cada partícula del universo hay contenido un mundo de infinitas criaturas"). Este no representaba un problema teórico para Leibniz, pero sí un problema práctico, es decir, para mostrar la consistencia y la utilidad de su enfoque.

\subsubsection{El cálculo leibniziano}

Como puede observarse, la aproximación leibniziana al cálculo es más bien algebraica. Leibniz fue ante todo un algebrista. Se esforzó por mostrarcómo muchos problemas pueden reducirse 
a sistemas de ecuaciones, los cuales descansan en última instancia en expresiones de la forma A es A. El principio de composicionalidad le lleva al atomismo, es decir, a sus mónadas (entendidas como totalidades "sin puertas ni ventanas"). Dentro de su visión de la filosofía, encontramos tres características principales del Universo: la plenitud, la continuidad y la degradación lineal. Esto permite formar una cadena de ser que, como señala Lovejoy, “... consiste de la totalidad de monadas, que van en orden jerárquico desde Dios al más bajo grado de vida sensible, no hay dos parecidas, sino que cada una difiere de las otras de abajo y de arriba de la escala por al menos una diferencia posible" (Lovejoy, 1933; citado en Wiener, 1951: xxxvi). El orden natural que deriva de estos tres principios presenta una armonía y una belleza que asombra a la razón. Orden en el que no hay vacíos, ni tampoco saltos.

En un ensayo publicado por Leibniz en 1715, titulado "Los fundamentos metafísicos de las matemáticas" muestra que las matemáticas son instanciaciones de estos principios generales, es decir, estos dominios las ponen de manifiesto: el espacio, el tiempo y el movimiento, siendo los dos primeros los básicos y el segundo el ámbito privilegiado de la física de su tiempo. Lo que es importante es que estos tres dominios muestran un nivel importante de isomorfismo. El mismo concepto de cantidad puede ser entendido en términos espaciales, temporales o de movimiento. En efecto, hablamos de cantidad para referirnos tanto a la extensión, a la duración como a la posición.

Otras propiedades importantes que comparten estos tres dominios son las siguientes: coexistencia para referirnos a eventos en el mismo espacio o límite espacial; sucesión para referirnos a eventos según la relación de orden de antecedenteconsecuente, y adyacencia para referirnos a las posiciones ocupadas sucesivamente por un objeto en movimiento. Estas propiedades, como puede verse, son también isomórficas.

Para ilustrar el hecho de que los principios de continuidad, plenitud y gradación lineal van más allá de las matemáticas, por lo menos de su tiempo, Leibniz considera la diferencia entre cantidad y cualidad. La cantidad, como hemos dicho, remite a la magnitud, sea esta espacial, temporal o de posición, mientras que la cualidad tiene que ver con las diferencias en las cualidades de los individuos o una especie considerados por sí mismos. Las cualidades constituyen algo así como los atributos fundamentales de un individuo o una especie. Cuando observamos cómo van variando las cualidades en el orden natural, encontramos una gradualidad progresiva a la cual podemos aplicar las mismas medidas matemáticas: la relación todo-parte, la relación mayor que, menor que, igual que, etc.

O como dice Leibniz, "la continuidad está presente en el tiempo tanto como en el proceso de la naturaleza debido a que ese proceso nunca tiene lugar por saltos repentinos" (p. 221). Comprender la naturaleza (como lo creado) es encontrar un orden construido a partir de estos principios generales y básicos, y ver cómo estos adquieren especificidades en diferentes dominios.

En este sentido, afirma Leibniz, una ecuación es un caso particular de la inecuación, el reposo es un caso particular del movimiento y las curvas son un caso particular de las líneas rectas. Debemos interpretar los infinitesimales como el esfuerzo que hacemos por convertir una serie infinita, es decir, una inecuación en una ecuación.

Dada su formación lógica, Leibniz elabora una notación para las integrales y para las derivadas que es la que se mantiene actualmente. En efecto, usa la " $\int$ ", es decir, una ese estilizada para referirse a la integral y la dx para la derivada. Esta notación se convirtió en la notación estándar a nivel continental.

\subsubsection{Continuación de Newton y Leibniz}

Durante y después de Newton y Leibniz, muchos prosiguieron sus investigaciones sobre el cálculo, tratando de darle consistencia al nuevo campo. Se crearon dos escuelas: la inglesa que siguió con la tradición newtoniana de las fluxiones y fluentes y la tradición continental liderada por Leibniz, los Bernoulli, Wallis, Fontenelle y una pléyade de destacados académicos. Será esta escuela la que alcanzará los mayores desarrollos en el área de las matemáticas. No es sino hasta 
el siglo XIX cuando decididamente se hacen los esfuerzos por introducir en Inglaterra la notación y los desarrollos matemáticos alcanzados a nivel del continente. Babbage, Hershel y Peacock, fundadores de la Sociedad Analítica, jugarán un importante papel aquí, al promover que se reemplazara la notación newtoniana por la leibniziana.

Ahora bien, Newton evitó discutir sobre la naturaleza de los infinitesimales, primos (primes) y razones últimas (ultimate ratios), utilizados en su método de análisis fluxional. Leibniz, por el contrario, hizo importantes esfuerzos por clarificar, sin éxito, la naturaleza de los infinitesimales.

Sin embargo, el siglo siguiente será, entre los ingleses, un siglo de cuestionamientos. Esto explica una parte del estancamiento del cálculo que encontramos en esta región. Mientras que estos trataban de resolver los problemas relacionados con el sentido de los conceptos de "infinitesimal", primos y razones últimas, más que preocuparse por el desarrollo del cálculo por sí mismo. Pero el otro factor es la relación ya mencionada en Newton de la relación entre el cálculo y la mecánica. Por ejemplo, Newton había partido de la existencia de espacio vacío, y por lo tanto, de la velocidad instantánea. Una de las identificaciones que encontramos aquí es entre la fluxión y el momentum. Pero también el uso de los infinitesimales en su paradójico uso: primero como incrementos y luego como decrecimientos, la división por cero, etc. fueron objeto de amplio debate.

En el continente encontramos, en contraste, que gracias a una fácil notación y a que muchos matemáticos entre ellos los Wallis, Bernouli y Fontanelle, hicieron grandes esfuerzos por popularizar el cálculo, la recepción del mismo resultara mucho más natural que en Inglaterra. Adicional a esto, el enfoque continental era más aritmético que geométrico. Esto hizo que en el continente fueran menos críticos que en Inglaterra, por lo tanto, que fueran menos críticos hacia la nueva disciplina matemática. Desde luego que no faltaron los críticos como Voltaire, quienes afirmaron que el nuevo cálculo es "el arte de numerar y medir una cosa cuya existencia no puede ser concebida".

\section{EULER}

Euler se encontraba así ante una serie de problemas. Primero, dos visiones antagónicas de la matemática: aquella que veía la matemática supeditada a la mecánica, de manera que el lenguaje de las matemáticas es el lenguaje de la mecánica. La otra que presentaba la matemática como un caso particular o instanciaciones de principios generales aplicables a todos los ámbitos del universo.

Segundo, no existía mayor claridad sobre cómo entender estos incrementos y decrementos, las diferencias entre las derivadas primeras y las de orden superior. Sobre esto Leibniz se ocupó en varias ocasiones, pero sin llegar a resultados convincentes. En algunos casos, sus planteamientos llevaban a confundir variables independientes de variables dependientes, etc. En otros, relacionados con la naturaleza de los infinitesimales, mantuvo tesis contrapuestas. Por un lado, afirmó que los infinitesimales eran ficciones, en otras ocasiones, mantuvo que eran ceros relativos, es decir, que el paso al límite no presenta los problemas de la división por cero.

Tercero, lo anterior hizo que el nuevo cálculo fuera sometido a una crítica devastadora, sobre todo por Berkeley. En su trabajo titulado The analyst. or a discourse addressed to an infidel mathematician, Berkeley critica el uso impreciso de los términos de incremento y decremento utilizados por Newton. Critica al matemático por apartarse del método propio de las matemáticas (la deducción) y proponer en su lugar una aproximación basada en métodos inductivos. Critica igualmente la identificación newtoniana del método de las fluxiones con el movimiento. De hecho, considera que la identificación de un incremento con el momento conlleva al problema de la asunción de la velocidad instantánea, la cual resulta problemática desde el punto de vista físico. Sin embargo, este punto no era tan problemático en su tiempo debido a que frecuentemente se suponía que la luz se desplaza de manera instantánea. Pero sí resulta problemática la interpretación que hace Newton del incremento como desvaneciéndose y convirtiéndose en cero, del cual ya hemos hablado. 
En este contexto podemos ubicar las contribuciones de Euler. Como todo intelectual no puede ir mucho más allá de las herramientas que le proporciona la época en la que le ha correspondido vivir. Euler se decide por la notación continental, debido claramente a la influencia que recibió de los hermanos Bernoulli. No cree en la concepción leibniziana de las mónadas o el atomismo matemático; lo rechaza por considerar que representa un uso muy abusivo del principio de razón suficiente. Representaría un mundo excesivamente poblado, al cual podríamos decir, deberíamos aplicar el principio de Ocán. Lo anterior significa que Euler conoce por tanto lo suficiente de la filosofía leibniziana, así como de la física newtoniana. Pero encuentra que la perspectiva continental ofrece mayores posibilidades de desarrollar el cálculo como un campo propio de las matemáticas. No solo promueve el uso de la notación continental, sino que introduce nueva notación para incorporarla a esa tradición la cual será usada a partir de ese momento. En este sentido, introduce el símbolo $e$ para referirse a los logaritmos neperianos, y el símbolo $\pi$ para denotar el número $3.14159 . .$. (aunque este último no fue primeramente usado por Euler), también utilizó $\mathrm{a}^{\mathrm{z}}$ para referirse a cualquier base del sistema logarítmico.

Euler sistematiza los desarrollos alcanzados hasta el momento en este campo. Pero lo que es más importante, hace importantes esfuerzos por liberar el cálculo de su dependencia de la mecánica y de la metafísica y desarrollarlo como un campo autónomo de las matemáticas. En este sentido escribió en 1748 una obra titulada Introductio in analysin infinitorum propuesta como una introducción a la matemática pura analítica. Esta obra se divide en dos partes. En la primera presenta un conjunto de resultados obtenidos hasta el momento, los cuales según Ball (1960) tienen la misma forma de los discutidos en los textos matemáticos modernos. La segunda parte tiene que ver con geometría analítica. Está dedicada al estudio de curvas bajo distintas formas de clasificación. Posteriormente, en 1755 publica su obra Instituciones calculi differentialis. En esta obra presenta una parte importante de los resultados personales alcanzados hasta el momento. Finalmente, en 1770 publica otra obra en alemán titulada Vollständige anleitung zur álgebra (algo así como "Instructivo completo sobre Álgebra") en dos volúmenes con diferentes resultados de su propia cosecha. Parece que fue Lagrange quien realizó una traducción al francés con nuevas adiciones por parte de Lagrange (Ball, 1960).

Hay dos contribuciones fundamentales realizadas por Euler que permitieron avanzar en la formalización y el desarrollo del cálculo infinitesimal en el siglo XIX.

La primera es haber formalizado el método infinitesimal en términos de funciones. Como señala Boyer (1959) el concepto de función fue introducido por Leibniz para indicar que el método infinitesimal no se limita a funciones algebraicas, sino también a los logaritmos y a los exponenciales. Pero, ciertamente, fue Euler quien adoptó la función de manera sistemática como la vía de formalizar el método infinitesimal, al parecer a solicitud de su amigo Daniel Bernoulli. Sin embargo, nos advierte Boyer que el concepto de función que utilizamos actualmente no fue el utilizado por Euler por carecer de los conocimientos modernos sobre el tema. Para él, la "palabra función representa (...) no tanto cualquier cantidad dependiente de variables, como una expresión analítica en constantes y variables que pueden ser representadas por símbolos individuales. Funcionalidad era un asunto de representación formal más que el reconocimiento conceptual de una relación. El desarrollo casi automático del cálculo durante el siglo dieciocho fue en gran parte el resultado de esta visión formalista, para la cual la notación de Leibniz era notablemente bien adaptada" (Boyer, 1961: 243). El enfoque funcional poco a poco reemplaza al análisis de las series infinitas que era la forma dominante de entender y aplicar los procedimientos del cálculo.

Esta aproximación funcional le permitió algo que Leibniz había iniciado: caracterizar conjuntos importantes de funciones y determinar algunas de sus propiedades. Esto tuvo un efecto importante: despegar el cálculo de funciones del marco geométrico en el que estaba inserto.

El segundo resultado importantísimo directamente relacionado con el anterior es el abandono de una concepción lógica, es decir, una concepción que preguntaba por la naturaleza de los 
incrementos o decrementos. Para los matemáticos del siglo XVIII lo mismo que para los filósofos, era fundamental entender qué tipo de entes eran estos. Por el contrario, con Euler se inicia un enfoque más orientado a la consistencia interna del sistema. En este sentido, Euler, nos dice Boyer, hizo explícito un punto de vista expresado por Leibniz que veía los infinitesimales como derivaciones de las cantidades finitas de partida. Esto siguiendo la ley de continuidad. Así, Euler considera la razón 0/0 (división por ceros) como "representando cualquier razón de números finitos n/1".

En este sentido debemos reconocer a Euler este paso fundamental en el despegue del cálculo como una teoría general de funciones.

\section{REFERENCIAS BIBLIOGRÁFICAS}

Ball, W. W. R. (1960). A short account of the history of mathematics. New York: Dover Publications Inc.

Boyer, C. (1959). The history of calculus. New York: Dover Publications Inc.

Buckley, M. J. (2003). El programa newtoniano y el origen del ateísmo. En Russell Stoeger \& Coyne. (2003). 97-122.

Cohen, B. \& Smith, G. (2002). Cambridge
Companion to Newton. Cambridge University Press.

Calinger, R. (1996). Leonhard Euler: the first St. Petersburg years (1727-1741). Historia Mathematica. 23 (2): 154-155.

Garber, D. \& Ayers, M. (1998). The Cambridge history of seventeeth-centure philosophy. Vol. 1. Cambridge University Press.

Hall, R. (2002). Newton versus Leibniz: from geometry to metaphysics. En Cohen \& Smith (2002). 431-454.

Leibniz (1715). Metaphysical foundations of mathematics. In: Wiener (1951). 201-216.

Russell, Stoeger \& Coyne. (2003). Física, filosofía y teología. Una búsqueda en común. México: Libros para todos.

Wiener, P. (1951). Leibniz selections. New York: Charles Scribner's Sons.

\section{SOBRE EL AUTOR}

\section{Celso Vargas Elizondo}

Doctor en Filosofía

Profesor catedrático de la Escuela de Ciencias Sociales del Instituto Tecnológico de Costa Rica Teléfono: (506) 25502281

Correo electrónico: celvargas@itcr.ac.cr 
